\title{
LXXV. The number of $\alpha$ particles emitted by uranium and thorium and by uranium minerals
}

\author{
Hans Geiger Ph.D \& Professor E. Rutherford F.R.S.
}

To cite this article: Hans Geiger Ph.D \& Professor E. Rutherford F.R.S. (1910) LXXV. The number of $\alpha$ particles emitted by uranium and thorium and by uranium minerals, Philosophical Magazine Series 6, 20:118, 691-698, DOI: 10.1080/14786441008636954

To link to this article: http://dx.doi.org/10.1080/14786441008636954

$$
\text { 曲 Published online: } 21 \text { Apr } 2009 .
$$

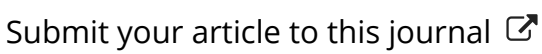

山 Article views: 17

Q View related articles $\sqsubset$

4 Citing articles: 15 View citing articles 
A relation between $\mathrm{P}_{n}\left(-\mu_{0}\right)$ and $\frac{d}{d \mu_{0}} \mathrm{P}_{n}\left(\mu_{0}\right)$ exists in this case, and allows us to put this result in the form

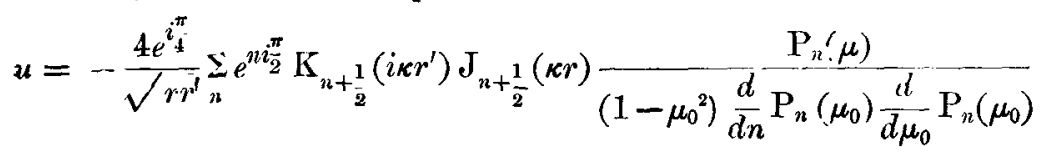

for $r<r^{\prime}$, the values of $n$ being as above.

The symbols $r, r^{\prime}$ have to be interchanged when $r>r^{\prime}$, and if the source were at $\left(r^{\prime}, \theta^{\prime}, \phi^{\prime}\right)$ instead of at $\left(r^{\prime}, 0,0\right)$, a corresponding, but more complicated, result would hold.

The problems in conduction of heat analogous to these lend themselves to the same treatment.

Sydney, June 1910.

LXXV. The Number of a Particles emitted by Uranium and Thorium and by Lranium Minerals. By Hans GeIger, Ph.D., and Professor E. Rutherford, F.R.S.*

I $\mathrm{N}$ previous papers we have shown that the number of 1. a particles emitted per second from radioactive materials can be counted either by the electrical or scintillation method. It has been shown that one gram of radium itself, and each of the three a ray products in equilibrium with it, emits $3.4 \times 10^{10} \propto$ particles per second. Since Rutherford and Boltwood $\dagger$ have shown that in an old unaltered mineral there is $3.4 \times 10^{-7}$ gram of radium per gram of uranium, it is possible to deduce the number of $\alpha$ particles emitted per second from one gram of uranium and also from a mineral containing one gram of uranium. In this calculation it is supposed that uranium is the ultimate parent of radium, and that the mineral is in radioactive equilibrium. If a uranium atom, like a radium atom, emits one a particle in its transformation, the number of a particles emitted per second per gram of uranium should be $3 \cdot 4 \times 10^{10} \times 3 \cdot 4 \times 10^{-7}$, or 11,600 . We shall for convenience call this number $\mathrm{N}$.

As a result of a very careful analysis of the radioactive constituents of uranium minerals, Boltwood $\ddagger$ has shown that the total activity of uraninm, measured by the electric method, is about twice as great as would be expected if

* Communicated by the Authors.

$\dagger$ Amer. Journ. Sci. vol. xxii. p. 2 (1906); also Boltwood, Amer. Journ. Sci. vol. xxv. p. 296 (1908).

$\ddagger$ Boltwood, Amer. Journ. Sci. vol. xxv. p. 270 (1908). 
uranium emits one $a$ particle for one from the radium itself in equilibrium with it. This suggests that the uranium atom in its transformation emits at least two $\alpha$ particles. In the present state of our knowledge it is not certain whether this can be ascribed to the existence of an additional $x$ ray product which is always separated with the uranium, or to the expulsion of two or more $\alpha$ particles in the transformation of the uranium atom.

Supposing, for the purpose of calculation, that the uranium in a mineral emits two $\alpha$ particles for one from each of the subsequent six $\alpha$ ray products, viz. ionium, radium emanation, radium $A$, radium $C$, radium $F$ (polonium), the number of a particles emitted per second per gram of uranium in a mineral is $8 \mathrm{~N}$, or four times the number emitted by ordinary purified uranium. In this calculation no account has been taken of the actinium which occurs in all uranium minerals, and which Boltwood has shown stands in a genetic relation with uranium. However, Boltwood (loc. cit.) has found that the actinium and its four $\alpha$ ray products contributes an activity to the mineral equal to only $\cdot 21$ of that of the uranium. The relative number of $\alpha$ particles is still smaller, for the $\alpha$ particles from actinium have an average range of about $5.7 \mathrm{cms}$. of air, while the a rays of uranium, according to Bragg, have a range of $3.5 \mathrm{cms}$. Taking as a first approximation that the ionization due to an a particle is proportional to its range, the number of $\alpha$ particles emitted by the actinium in a mineral should be about 17 of that from uranium. The total number of $\alpha$ particles emitted by a mineral containing one gram of uranium should consequently be $2 \cdot 34 \mathrm{~N}+6 \mathrm{~N}=8 \cdot 34 \mathrm{~N}$. Since $\mathrm{N}$ by calculation is 11,600 , the total number of a particles emitted per second from a mineral containing one gram of uranium should be $9 \cdot 67 \times 10^{4}$, and the number per second from one gram of ordinary purified uranium should be $2.32 \times 10^{4}$.

It was the object of the present experiments * to determine the number of $\alpha$ particles experimentally, and to test the agreement with the calculated number.

* The experiments described later were, for the most part, completed more than a year ago. Recently, J. N. Brown (Proc. Roy. Soc. vol. A. lxxxiv. p. 151, 1910) has counted the scintillations from a uranium mineral and found a value per gram of uranium of $7.36 \times 10^{4}$, which is somewhat smaller than our experimental value given later, viz. $8.6 \times 10^{4}$ 
Arrangement of Experiment.

The scintillation method was adopted in order to count the number of a particles from a known weight of active material. A small quantity of the material under examination was finely powdered in an agate mortar, and then mixed with alcohol or ether and deposited as a thin uniform film on a thin sheet of aluminium or glass. The method adopted was similar to that first used by McCoy. Care was taken that the powder suspended in the liquid was well stirred in order to avoid a separation of the lighter from the denser portions. The weight of the active film was determined by weighing the plate before and after the active material had been removed. It was desirable to use very thin films in order that all the a particles might emerge without much loss of their range. In the case of uranium, however, the number of $\alpha$ particles emitted was so small that they were difficult to count with accuracy. For this reason thicker films were in some cases purposely employed. The efficiency of the zinc sulphide screen was tested by counting the number of a particles emitted from a definite quantity of radium C. The number of scintillations observed was found to be 8 per cent. less than the actual number of $\alpha$ particles incident on the screen. The latter value was calculated from the known result that one gram of radium and each of its products enits $3.4 \times 10^{10} \propto$ particles per second. In the initial experiments the number of scintillations was counted by placing the screen close to the active material. In this case, the number of $\alpha$ particles striking the screen is equal to one half the total number emitted from an area of the active film equal to the area of screen seen in the microscope. This method is open to some objections, for it requires that the film should be very uniformly spread and, in addition, very thin, for otherwise the particles emitted at an oblique angle suffer a considerable loss of range in the active material itself. The lack of uniformity of the film can be corrected for by counting at different points parts of the film, but this involves much labour.

In most of the experiments the active matter was spread in a circular area, and the small zinc sulphide screen was placed parallel to the film and opposite to its centre.

If $\quad a=$ radius of circular film,

$d=$ distance of screen from centre of film,

$\mathrm{A}=$ area of screen observed in field of microscope, $\sigma=$ total number of particles emitted per second per square centimetre of surface of film, 
694 Dr. Geiger and Prof. Rutherford on the Number of

then, by a simple integration, it can be shown that the number $n$ of $\alpha$ particles incident per second on the area $A$ is given by

$$
n=\frac{\sigma \mathrm{A}}{2}\left(1-\frac{d}{\sqrt{a^{2}+d^{2}}}\right) .
$$

A simple example will serve to illustrate the method of calculation. The uranium film No. 1 (see table later) contained 10.43 milligrams of uranium oxide $\left(\mathrm{U}_{3} \mathrm{O}_{8}\right)$ spread on an area of 5.9 square cms. 515 scintillations were counted, and the average number of scintillations observed corresponded to $5 \cdot 16$ per minute, and per second $\cdot 086$. Making the 8 per cent. correction for the imperfection of the screen, the corrected value becomes $\cdot 093$. This is the value of $n$ to be substituted in the formula.

$$
\mathrm{A}=3.16 \text { sq. mms. } \quad d=2.06 \mathrm{cms} . \quad a=1.37 \mathrm{cms} \text {. }
$$

Substituting these values in the formula,

$$
\sigma=35 \cdot 0 \text {. }
$$

Now the weight of film per square centimetre was $1.77 \mathrm{mg}$. $\mathrm{U}_{3} \mathrm{O}_{8}$, or $1.50 \mathrm{mg}$. uranium. Consequently, from this experiment, the total number of a particles emitted per second per gram of uranium is $2 \cdot 33 \times 10^{4}$.

The chief difficulty of the experiments lay in counting accurately a sufficiently large number of scintillations. The numbe: of scintillations observed in the microscope varied from one to tive per minute in the case of uranium or thorium. While different observers agreed closely in counting scintillations due to radium or polonium when 30 to 50 scintillations were seen per minute, the agreement was not so good for uranium films. This difference is in part due to the fact that the eye becomes quickly fatigued when only a few scintillations appear on the screen per minute. This was especially marked in counting the scintillations from uranium, which are relatively much fainter than those from radium (y. In the case of uranium and thorium minerals, where the scintillations are on the average much brighter than those from uranium, the counting was relatively easy. The brightness of scintillations of course depends on the range of the $\alpha$ particle striking the screen. We shall see later that the range of the $\alpha$ particle, and consequently the intensity of the scintillations from uranium, is less than from any other radioactive substance.

The active materials used in these investigations were 
a Particles emitted by Uranium and Thorium.

kindly presented to us by Professor Boltwood, and were fractions of larger quantities analysed by him. We desire to express our indebtedness to Professor Boltwood for the use of these materials.

(1) Uranic-uranose oxide $\left(\mathrm{U}_{3} \mathrm{O}_{8}\right)$ prepared from uranium nitrate which had been crystallized fifteen times. The least soluble fraction was taken and ignited at a high heat in a current of oxygen.

(2) Uraninite-a selected sample from Joachimsthal. This contained 61.7 per cent. of uranium. The mineral, when finely powdered, lost $6 \cdot 2$ per cent. of its emanation. The sample employed had been finely ground for several years, and during this time the emanation had steadily escaped. Under these conditions it can be simply deduced that the emission of a particles from the mineral is about three per cent. less than if the mineral had retained all its emanation. A correction of this amount has consequently been made to the counted number of a particles.

(3) Thorium oxide prepared from thorite. This was tested five weeks after its chemical separation. Since, in the chemical process of purification, the mesothorium is removed from the thorium, the a-ray activity of the purified thorium decays with time due to the decay of its product radiothorium. Since the half period of decay of the latter is about 737 days, a positive correction of about two per cent. is necessary to give the correct number of $\alpha$ particles emitted from thorium oxide in radioactive equilibrium. The activity of the thorium oxide in the form of a thin film was compared with that of a film of the mineral thorite of known composition, and gave nearly the ratio to be expected from their relative content of thorium.

The results of the observations are included in the following Table (p. 696).

Since only about 900 scintillations were counted altogether, the agreement between the three uranium films is closer than could be expected, considering the possible errors in the experiment. In the case of the mineral films 2000 scintillations were counted in all, and about an equal number for the thorium films. Before and after each set of observations the screen was carefully tested to determine the number of scintillations observed when the active material was removed. The correction for the screen employed was small, and usually corresponded to one scintillation in three or four minutes. All the counting experiments were checked among themselves by measuring the activity of the films in an $\alpha$-ray electroscope. The activity measured in this way was found 
Dr. Geiger and Prof. Rutherford on the Number of

to be proportional to the weight of the film for thin films, but for the thicker films the activity was relatively smaller on account of absorption.

\begin{tabular}{|c|c|c|}
\hline Radioactire Substances. & \multicolumn{2}{|c|}{$\begin{array}{l}\text { Number of } \propto \text { particles emitted } \\
\text { per second per gram of } \\
\text { Uranium or Thorium. }\end{array}$} \\
\hline $\begin{array}{l}\text { Uranium film No. } 1 . \\
10.43 \mathrm{mgrs} . U_{3} \mathrm{O}_{8} \text { on area } 5.9 \mathrm{~cm} .^{2}\end{array}$ & $2.33 \times 10^{4}$ & \\
\hline $\begin{array}{l}\text { Uranium film No. } 2 . \\
285 \text { mgrs. } \mathrm{U}_{3} \mathrm{O}_{8} \text { on area } 128 \mathrm{~cm}^{2}\end{array}$ & $236 \times 10^{4}$ & $\begin{array}{c}\text { Average } \\
2 \cdot 37 \times 10^{4}\end{array}$ \\
\hline $\begin{array}{l}\text { Uranium film No. } 3 . \\
3.04 \text { mgrs. } \mathrm{U}_{3} \mathrm{O}_{8} \text { on area } 14.9 \mathrm{~cm} .^{2}\end{array}$ & $2 \cdot 43 \times 10^{1}$ & \\
\hline $\begin{array}{c}\text { Mineral film No. } 1 . \\
10.95 \text { mgrs. Uraninite. } \\
\text { ("Foachimstahl) on area } 5.9 \mathrm{~cm}^{2}{ }^{2}\end{array}$ & $95 \times 10^{4}$ & \\
\hline $\begin{array}{c}\text { Mineral film No. } 2 . \\
12 \cdot 73 \mathrm{mgrs} \text {. Uraninite. } \\
\text { (Joachimstahl) on area } 5.9 \mathrm{~cm} .^{2}\end{array}$ & $9 \cdot 7 \times 10^{4}$ & $96 \times 10^{2}$ \\
\hline $\begin{array}{l}\text { Thorium film No. } 1 . \\
4 \cdot 43 \text { mgrs. } \mathrm{ThO}_{2} \text { on } 6 \cdot 1 \mathrm{~cm} .^{2}\end{array}$ & $255 \times 10^{1}$ & \\
\hline $\begin{array}{l}\text { Thorium film No. } 2 . \\
1.21 \text { mgrs. ThO } \mathrm{O}_{2} \text { on } 6.4 \mathrm{~cm} .^{2}\end{array}$ & $2 \cdot 84 \times 10^{1}$ & $\begin{array}{l}\text { A verage } \\
27 \times 10^{4}\end{array}$ \\
\hline $\begin{array}{l}\text { Thorium film No. } 3 . \\
3.58 \text { mgrs. } \mathrm{ThO}_{2} \text { on } 6.15 \mathrm{~cm}^{2}\end{array}$ & $2.65 \times 10^{t}$ & \\
\hline
\end{tabular}

It will be seen that there is a good agreement between the experiments and the numbers calculated on the assumption considered in the beginning of this paper. This is brought out by the Table below.

Number of a particles per gram of Uranium per second.

Calculated.

Uranium

$2 \cdot 32 \times 10^{4}$

Observed.

Uranium mineral

$9.67 \times 10^{4}$

$2 \cdot 37 \times 10^{4}$

Thorium, number of $\propto$ particles per gram : $2 \cdot 7 \times 10^{4}$

No doubt the agreement is closer than would be expected under the conditions of the experiments. 
The agreement between theory and experiment confirms in another way the correctness of Boltwood's conclusion that uranium emits two a particles for one from each of its later products. The experiments are not of sufficient accuracy to confirm the data on the relative activity of actinium and radium. There is no doubt, however, that the number of * particles to be ascribed to actinium is very small compared with that to be expected if actinium and its series of products emitted one $\alpha$ particle for one from radium. The connexion of actinium with the uranium-radium series is difficult to determine, and remains one of the chief outstanding problems in the analysis of radioactive changes.

\section{Production of Helium by Uranium, Uranium Minerals, and 'Lhorium.}

Since the $\alpha$ particle is a charged atom of helium, it is a simple matter to deduce the rate of production of helium from the active materials considered. Calculation and experiment show that one gram of radium in equilibrium with its three $\alpha$-ray products produces 158 cubic $\mathrm{mm}$. of helium per year. Since radium and each of its products emits $3.4 \times 10^{10}$ a particles per gram per second, uranium, which emits $2.37 \times 10^{4} \alpha$ particles per gram per second, produces $2.75 \times 10^{-5}$ cubic $\mathrm{mm}$. per year. The rate of production of helium for the different materials is given below.

\begin{tabular}{|c|c|c|}
\hline & $\begin{array}{r}\text { Production } \\
\text { per gram } \\
2 \cdot 75 \times 10^{-5}\end{array}$ & $\begin{array}{l}\text { elium } \\
\text { ear. } \\
\text { c mm. }\end{array}$ \\
\hline Thorium ......... & $3 \cdot 1 \times 10^{-5}$ &, \\
\hline $\begin{array}{l}\text { Urinium mineral in equi- } \\
\text { librium .................... }\end{array}$ & $11 \cdot 0 \times 10^{-5}$ & $"$ \\
\hline Radium in equilibrinm & 158 & , \\
\hline
\end{tabular}

A simple calculation allows us to estimate the production of helium for a mineral like thorianite containing both uranium and thorium.

\section{Range of the a particles from Uranium.}

The range of the $\alpha$ particles from uranium has been difficult to determine directly on account of the smallness of the activity of the thin films of the substance. By observations of the decrease of the ionization due to a layer of uranium when sheets of thin aluminium were placed over it, Bragg * deduced that the range in air of the $\alpha$ particle from uranium was about $3.5 \mathrm{cms}$. In the course of counting the scintillations from a thin film of ionium, it was observed that 
the scintillations were as bright if not brighter than those from a thin film of uranium. Boltwood has found that the range of the $a$ particle from ionium is $2.8 \mathrm{cms}$., so that it appeared probable that the range of the $\alpha$ particles from uranium had been overestimated. This conclusion was confirmed by finding that the a rays from a thin film of uranium were more readily absorbed by aluminium than those from ionium. By a special method, the range of the $\alpha$ particle from uranium has been measured and found to be about $2.7 \mathrm{cms}$, while the range of the $\alpha$ particle from ionium is a millimetre or two longer. Further experiments are in progress to determine the range of the $\alpha$ particle from uranium accurately, and to examine carefully whether two sets of $\alpha$ particles of different range can be detected.

University of Manchester, July 1910.

LXXVI. The Probability Variations in the Distribution of a Particles. By Professor E. Rutherford, F.R.S., and H. Geiger, Ph.D. With a Note by H. Bateman *

TN counting the a particles emitted from radioactive 1 substances either by the scintillation or electric method, it is observed that, while the average number of particles from a steady source is nearly constant, when a large number is counted, the number appearing in a given short interval is subject to wide fluctuations. These variations are especially noticeable when only a few scintillations appear per minute. For example, during a considerable interval it may happen that no $\alpha$ particle appears; then follows a group of $\alpha$ particles in rapid succession ; then an occasional $\alpha$ particle, and so on. It is of importance to settle whether these variations in distribution are in agreement with the laws of probability, $i$. $e$. whether the distribution of $\alpha$ particles on an average is that to be anticipated if the $\alpha$ particles are expelled at random both in regard to space and time. It might be conceived, for example, that the emission of an $\alpha$ particle might precipitate the disintegration of neighbouring atoms, and so lead to a distribution of $\alpha$ particles at variance with the simple probability law.

The magnitude of the probability variations in the number of $a$ particles was first drawn attention to by E.v. Schweidler $\dagger$. He showed that the average error from the mean number of a particles was $\sqrt{\mathrm{N} . t}$, where $\mathrm{N}$ was the number of particles emitted per second and $t$ the interval under consideration. This conclusion has been experimentally verified by several

- Communicated by the Authors.

† v. Schweidler, Congrès Internationale de Radiologie, Liègee, 1905. 